\title{
Índice interno de variação de preços de materiais de consumo e de medicamentos em um hospital de ensino de alta complexidade
}

\author{
Internal rate of varying prices of consumable and medicines in a \\ high complexity university hospital
}

Denise Cristina Carraro ${ }^{1}$, Maria Eulália Lessa do Valle Dallora²

\begin{abstract}
RESUMO
Modelo do estudo: Trata-se de um estudo de caso, no qual foram analisados dados dentro de um contexto real que é o do HCFMRP-USP Objetivos: Apresentar os índices de variação de preços do HCFMRP-USP (IVPH), ano 2013, geral para materiais de consumo e medicamentos; específico para materiais de consumo e específico para medicamentos. Materiais e Método: Para o IVPH foi definido o Índice de Paasche como o mais adequado para o perfil de consumo do Hospital. Para o cálculo, foi definida uma cesta de consumo considerando $40 \%$ do gasto financeiro em 2012 o que representou 13\% dos itens e também pelos itens que tiveram consumo regular, ou seja, em todos os meses de janeiro a dezembro de 2013. Resultados: os IVPH gerais foram $6,74 \%$ para materiais e medicamentos; 6,97\% para materiais de consumo e 6,73\% para medicamentos. Conclusões: Comprar a preços praticados no mercado e utilizar ferramentas para analisar o poder de compra da instituição contribui para a aplicação correta e racional dos parcos recursos disponíveis. A proposta de criar o índice interno de variação de preços vem contribuir como ferramenta para gestão dos recursos e para a análise da evolução do poder de compra do HCFMRP-USP, assim como os índices padronizados de variação de preço são utilizados para análise do poder de compra da moeda.
\end{abstract}

Palavras-chave: Inflação. Administração Hospitalar. Economia Hospitalar. Administração de Serviços de Saúde. Economia.

\begin{abstract}
Study model: This is a case study in which data from a real hospital context were analyzed. Objectives: To describe the price variation indexes of HCFMRP-USP (IVPH), in the year 2013, general for consumer materials and medicines, specific for consumer and specific for drugs.
\end{abstract}

1. Assessora Técnica III da Assessoria Técnica do Hospital das Clínicas da Faculdade de Medicina de Ribeirão Preto da Universidade de São Paulo.

2. Dirigente da Assessoria Técnica no Hospital das Clínicas da Faculdade de Medicina de Ribeirão Preto da Universidade de São Paulo.
CorRespondÊnCIA: Denise Cristina Carraro Assessoria Técnica Hospital das Clínicas da Faculdade de Medicina de Ribeirão Preto da USP CEP 14048-900, Ribeirão Preto - SP

Recebido em 18/01/2017 Aprovado em 27/02/2018 
Methods: For the IVPH, the Paasche Index was defined as the most adequate for the hospital consumption. For the calculation, a consumption set was defined as $40 \%$ of the financial expense in 2012 , which represented $13 \%$ of the items and alse the items that had regular consumption, that is, in all the months of January to December 2013. Results: The general IVPH were $6.74 \%$ for materials and medicines; $6.97 \%$ for consumer materials and $6.73 \%$ for medicines. Conclusions: Buying at prices practiced in the market and using tools to analyze the purchasing power of institution contributes to the correct and rational application of the meager resources available. The proposal to create the internal price variation index is to contribute as a resource management tool and to analyze the evolution of purchasing power of the HCFMRP-USP, as well as the standardized index of price variation is used to analyze the power of purchase of the currency.

Key words: Inflation. Hospital Administration. Economics, Hospital. Health Services Administration. Economics.

\section{INTRODUÇÃO}

A busca de equilíbrio econômico-financeiro em instituições de saúde, sejam elas públicas ou privadas, vem se constituindo em grande desafio para as suas sobrevivências. ${ }^{1}$

Equacionar os recursos disponíveis para o custeio das atividades frente à pressão exercida pelas demandas, garantindo a qualidade da assistência prestada torna-se um dilema e um grande desafio para os gestores dos recursos públicos. ${ }^{2}$

O aumento dos gastos em saúde é ocasionado por diversos fatores que agem sobre a demanda por serviços de saúde, dentre eles, o aumento da cobertura, o aumento dos preços dos bens e serviços, o envelhecimento da população, a transição epidemiológica com aumento de complexidade de casos, bem como o aparecimento de novas doenças, a incorporação tecnológica com o advento da introdução de novos equipamentos, medicamentos, próteses, órteses e outros, o esclarecimento populacional: a população mais esclarecida reivindica seus direitos. ${ }^{3-9}$

Em economias abertas, como é o caso do Brasil a partir da década de 90 , nas quais as relações como o exterior são de fundamental importância, uma grande influência no aumento de gastos é a da chamada inflação importada. Quando ocorre um aumento de preços nos mercados internacionais, relativo a um vasto conjunto de bens, ou a um único produto, mas de difícil substituição, isso pode refletir no nível de preços interno dos países importadores. A situação poderá ainda agravar-se, quando se trata de um produto utilizado como input em múltiplos ramos de atividade. Este é o caso do setor saúde, altamente dependente de produtos e insumos do mercado internacional. $5,8,9,10$

A política cambial também pode ser causa de inflação. Assim, se a moeda nacional sofrer uma desvalorização, isso resultará num encarecimento das importações: o mesmo preço em moeda estrangeira, dólares, euros ou ienes, por exemplo, traduz-se num preço mais alto em moeda nacional, pois se torna necessária maior quantidade desta para adquirir a mesma quantidade de divisas estrangeiras. Logo, ou o volume de importações diminui, ou os preços internos aumentam ou sucede um pouco de ambas as coisas. ${ }^{9}$

O financiamento tem se constituído numa preocupação permanente dos gestores e de todos os envolvidos diretamente com a construção do sistema de saúde, tornando-se tema constante em discussões das instâncias gestoras. Para - SUS, garantir a universalidade e integralidade diante de um cenário de restrições orçamentárias e financeiras e alocar recursos de forma equânime num país de tantas desigualdades sociais e regionais tem se transformado num grande desafio para os gestores. ${ }^{11,12}$

Globalmente, existe uma discrepância entre as necessidades de financiamento da saúde dos países e de suas despesas de saúde. Os países em desenvolvimento respondem por $84 \%$ da população mundial e $90 \%$ da carga global de doença, mas apenas $12 \%$ dos gastos em saúde global. Os países mais pobres suportam uma parcela ainda maior da carga de doenças e lesões, mas eles têm menos recursos para o financiamento de serviços de saúde. ${ }^{12,13,14}$ 
Planejar estrategicamente, a médio e longo prazo, observando cenários do desenvolvimento econômico são elementos que propiciam ao país, um sistema de saúde desejado. ${ }^{5}$

A gestão dos recursos em saúde sofre impacto da variação de preços, especialmente quando há inflação e os problemas decorrentes desta situação são vários. ${ }^{15}$

No ambiente de empresas, criar o indicador interno de variação de preços possibilita obter comparações com os diversos índices oficiais no país e contribui para o planejamento, avaliação dos resultados e para a gestão estratégica. Quando a variação interna de preços é maior que zero temos a inflação interna da empresa. ${ }^{16}$

A inflação interna pode também ser influenciada pelo impacto do aumento no volume de drogas dispensadas para cada paciente internado que está recebendo tratamento ou para cada tratamento ambulatorial. Quanto maior o número de dias de tratamento maior o impacto do volume de drogas utilizadas. ${ }^{16,17}$

As variáveis citadas acima devem ser gerenciadas pelos gestores de sistemas de saúde que tem de considerá-las no processo de operacionalização do sistema e, se não forem bem administradas podem inviabilizar a prestação de serviços visto que as receitas/financiamentos não crescem na mesma proporção. $8,10,18$

O índice de variação de preços tem o propósito de contribuir para a negociação orçamentária e dimensionamento do volume das atividades desenvolvidas. Assim, colaborar com a meIhoria da capacidade de planejamento das ações e recursos destinados ao custeio de materiais e medicamentos.

No Hospital das Clínicas da Faculdade de Medicina de Ribeirão Preto da Universidade de São Paulo (HCFMRP-USP) essa situação é também vivenciada. Assim, este trabalho tem como objetivos apresentar os índices de variação de preços do HCFMRP-USP (IVPH) para materiais de consumo e medicamentos; específico para materiais de consumo e específico para medicamentos.

\section{Índices de variação de preços}

Vários tipos de índices são utilizados para o cálculo das variações de preços. ${ }^{19,20,21}$
Os índices de preços são utilizados para monitorar mudanças nos níveis de preços ao longo do tempo. Os dois mais comuns são o Índice de Laspeyres, criado por Etienne Laspeyres e o Índice de Paasche, criado por Hermann Paasche. ${ }^{21}$

Os índices de preços de Laspeyres e de Paasche geram, em geral, resultados diferentes quando utilizados para avaliar a variação do nível dos preços de um conjunto de produtos, mas ambos atendem à condição de reversão no tempo. ${ }^{20}$

O índice de Laspeyres considera que os recursos de um indivíduo devem ser os mesmos para a mesma cesta de bens no período corrente como no período base. Mantidas as quantidades, os preços podem variar ou não. A ponderação é feita em função do período base ou inicial. ${ }^{21,22}$

A fórmula a seguir representa o índice de variação de preços de Laspeyres.

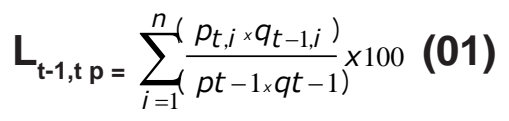

Onde:

$\mathrm{p}_{\mathrm{t}, \mathrm{i}}=$ preço do item i no período $\mathrm{t}$

$\mathrm{p}_{\mathrm{t}-1, \mathrm{i}}=$ preço do item i no período $\mathrm{t}-1$, imediatamente anterior

$q_{t-1, i}=$ quantidade do item i no período $t-1$, imediatamente anterior

$\mathrm{t}=$ período de referência

$\mathrm{t}-1$ = período imediatamente anterior

No índice de Paasche, a ponderação é feita em função do período de referência. Por este motivo é tradicionalmente utilizado no cálculo dos índices de preços. ${ }^{21,23,24}$

$$
\mathbf{P}_{\mathrm{t}-\mathbf{1}, \mathbf{t}}=\sum_{i=1}^{n} \frac{\left(p_{t, i} \times q_{t, i}\right)}{\left.p_{t-1, i} \times q_{t, i}\right)} \times 100
$$

Onde:

$\mathrm{p}_{\mathrm{t}, \mathrm{i}}=$ preço do item i no período $\mathrm{t}$

$\mathrm{p}_{\mathrm{t}-1, \mathrm{i}}=$ preço do item i no período $\mathrm{t}-1$, imediatamente anterior

$$
\begin{aligned}
& \mathrm{q}_{\mathrm{t}, \mathrm{i}}=\text { quantidade do item i no período } \mathrm{t} \\
& \mathrm{t}=\text { período de referência }
\end{aligned}
$$

No Brasil dentre os índices de variação de preços mais conhecidos temos os descritos a se- 
guir, com as respectivas instituições responsáveis pelos cálculos:

- Índice Nacional de Preços ao Consumidor (INPC), do Instituto Brasileiro de Geografia e Estatística - IBGE;

- Índice Geral de Preços (IGP), da Fundação Getúlio Vargas - FGV;

- Índice Geral de Preços - Disponibilidade Interna (IGP-DI), da Fundação Getúlio Vargas - FGV;

- Índice de Preços ao Consumidor (IPC), da Fundação Instituto de Pesquisas Econômicas - FIPE;

- Índice de Preços ao Consumidor Ampliado (IPCA), do Instituto Brasileiro de Geografia e Estatística - IBGE;

- Índice de Custo de Vida (ICV), do Departamento Intersindical de Estatísticas e Estudos Socioeconômicos - DIEESE.

O INPC é medido pelo IBGE desde setembro de 1979. É obtido a partir dos Índices de Preços ao Consumidor regionais e tem como objetivo oferecer a variação dos preços no mercado varejista, mostrando, assim, o aumento do custo de vida da população. ${ }^{23}$

O IGP que é medido pela FGV, registra a inflação de preços desde matérias-primas agrícolas e industriais até bens e serviços finais. É divulgado em três versões: IGP-DI, Índice Geral de Preços do Mercado (IGP-M) e Índice Geral de Preços 10 (IGP-10). O que difere as três é o período de pesquisa dos dados.

O IGP-DI é medido pela FGV e registra a inflação de preços desde matérias-primas agrícolas e industriais até bens e serviços finais. Faz medições no mês cheio, de 1 a 30 ou 31 de cada mês. Abrange toda a população, sem restrição de nível de renda. É utilizado para reajustes de tarifas públicas, contratos de aluguel e planos e seguros de saúde, nos contratos mais antigos. ${ }^{20,25}$

O IPC-FIPE é calculado medindo-se o mês cheio, de 1 a 30 ou 31, e de maneira quadrissemanal. O sistema de cálculo da variação quadrissemanal do IPC-Fipe abrange um período de oito semanas de coleta. Em cada quadrissemana, as variações são obtidas dividindo-se os preços médios das quatro últimas semanas referência pelos preços médios das quatro semanas anteriores a elas (base). ${ }^{25}$
O IPCA - Índice de Preços ao Consumidor Amplo, medido mensalmente pelo IBGE, foi criado com o objetivo de oferecer a variação dos preços no comércio para o público final e medir as variações de preços referentes ao consumo pessoal. O IPCA é considerado o índice oficial de inflação do país. O período de coleta do IPCA vai do dia 1ㅇ ao dia 30 ou 31, dependendo do mês. ${ }^{23,25}$

O ICV elaborado pelo DIEESE é calculado em três extratos distintos: Extrato 1 - famílias com menor renda, um a três salários mínimos; Extrato 2 - Famílias com renda intermediária, um a cinco salários mínimos e Extrato 3 - Famílias de maior poder aquisitivo, um a 30 salários mínimos. Mede a variação do custo de vida das famílias com renda de um a 30 salários mínimos do município de São Paulo. Para obter o ICV, o DIEESE aplica a fórmula de Laspeyres. ${ }^{12,25}$

\section{MATERIAIS E MÉTODOS}

Para o IVPH foi definido o Índice de Paasche como o mais adequado para o perfil de consumo do HCFMRP-USP por ser este o índice que considera a ponderação ou peso dos itens em função dos preços e quantidades do período de referência. Este estudo considerou 2013 o período de referência. Para se obter o IVPH de 2013, houve necessidade de se obter as quantidades e valores dos materiais de consumo e medicamentos adquiridos em 2012 para definir a cesta de consumo.

Com a cesta definida, obteve-se o levantamento das compras dos itens, no ano de 2013, mês a mês, desde que tenham tido consumo regular, ou seja, quantidades consumidas em todos os meses. Portanto, foram excluídos os itens com consumo zero em qualquer mês de 2013. Os preços considerados foram os últimos pagos. No levantamento foram consideradas as seguintes informações:

- Código do Material

- Nome do Material

- Nome do grupo de material (gêneros alimentícios, material didático, médico hospitalar, etc)

- Unidade do material - especificação da unidade de compra (cento, resma, etc.).

- Últimos preços pagos

- Quantidades consumidas 
Um fator limitante neste estudo diz respeito aos materiais órteses e próteses que apresentaram falhas de registros de saída de estoque no sistema de gestão de materiais. Devido a isto, diversos itens de órteses e próteses foram excluídos deste estudo.

Base de cálculo do índice de variação de preços de materiais de consumo e medicamentos do HCFMRP-USP

No numerador tem-se os valores do período de referência tanto para preço quanto para quantidade e, no denominador tem-se o valor do período imediatamente anterior para preço e o valor do período de referência para quantidade.

$$
\mathrm{IVPH}_{\mathrm{t}-1, \mathrm{t}}=\sum_{i=1}^{n} \frac{\left(p_{t, i} \times q_{t, i}\right)}{\left.p_{t-1, i} \times q_{t, i}\right)} \times 100
$$

Onde:

$p_{t, i}=$ preço do item i no período $t$

$\mathrm{q}_{\mathrm{t}, \mathrm{i}}=$ quantidade do item i no período $\mathrm{t}$

$\mathrm{p}_{\mathrm{t}-1, \mathrm{i}}=$ preço do item i no período $\mathrm{t}-1$

$\mathrm{q}_{\mathrm{t}-1, \mathrm{i}}=$ quantidade do item i no período $\mathrm{t}-1$

$\mathrm{t}=$ período de referência

$\mathrm{t}-1$ = período imediatamente anterior

\section{Definição da cesta de consumo}

Considerou-se os itens que apresentaram consumo regular em 2012 ou seja, consumo em todos os meses. Uma planilha eletrônica foi gerada com colunas representando os meses do ano de 2012 e linhas representando os itens da cesta de consumo. Em cada célula da planilha constou a quantidade consumida do item multiplicado pelo último preço pago no mês.

O corte nesta planilha, que continha 12.096 itens, foi realizado em $40 \%$ do valor financeiro total o que representou $13 \%$ da quantidade de itens. Considerou-se adequado o corte de 40-13 para definir os itens de composição da cesta, pois estes eram os que apresentavam maiores pesos (preço $\times$ quantidade).

\section{Cálculo da variação de preços}

A partir da obtenção da cesta de consumo, adotando o conceito do índice de Paasche, para o cálculo da variação de preços, foram utilizados os dados das quantidades consumidas mês a mês, em 2013 de cada item e os últimos preços pagos em cada mês, desde que houvesse consumo regular.

\section{RESULTADOS}

\section{Índice de variação de preços geral para} materiais de consumo e medicamentos do HCFMRPUSP no ano de 2013

O índice de variação de preços para materiais de consumo e medicamentos obtido no ano de 2013 foi $6,74 \%$.

Este resultado caracteriza inflação acumulada no ano de 2013, pois houve aumento geral de preços para materiais de consumo e medicamentos.

Os itens que tiveram maiores contribuições na variação dos preços foram: Anfotericina B lipossomal injetável fr $50 \mathrm{mg}$, com contribuição de 5,43\%; Voriconazol comprimido $200 \mathrm{mg}$, com $3,90 \%$ e I matinib comprimido $400 \mathrm{mg}$, com 3,87\%.

Índices específicos de variações de preços para materiais de consumo do HCFMRP USP no ano de 2013

O índice de variação de preços específico para materiais de consumo obtido em 2013 foi $6,97 \%$.

Este resultado caracteriza inflação acumulada no ano, pois houve aumento geral de preços para materiais de consumo. Os itens que tiveram maiores contribuições na variação dos preços foram: o óleo combustível BPF-1, com 4,03\% de contribuição; luva de látex, com 3,07\% e papel toalha, com $2,80 \%$.

Os IVPH estratificados por grupo de material de consumo seguem demonstrados no Gráfico 1 .

Índices específicos de variações de preços para medicamentos do HCFMRP USP no ano de 2013

O índice de variação de preços específico para medicamentos em 2013 foi 6,73\%. Este resultado caracteriza inflação acumulada no ano de 2013, pois houve aumento geral de preços para materiais de consumo e medicamentos. 


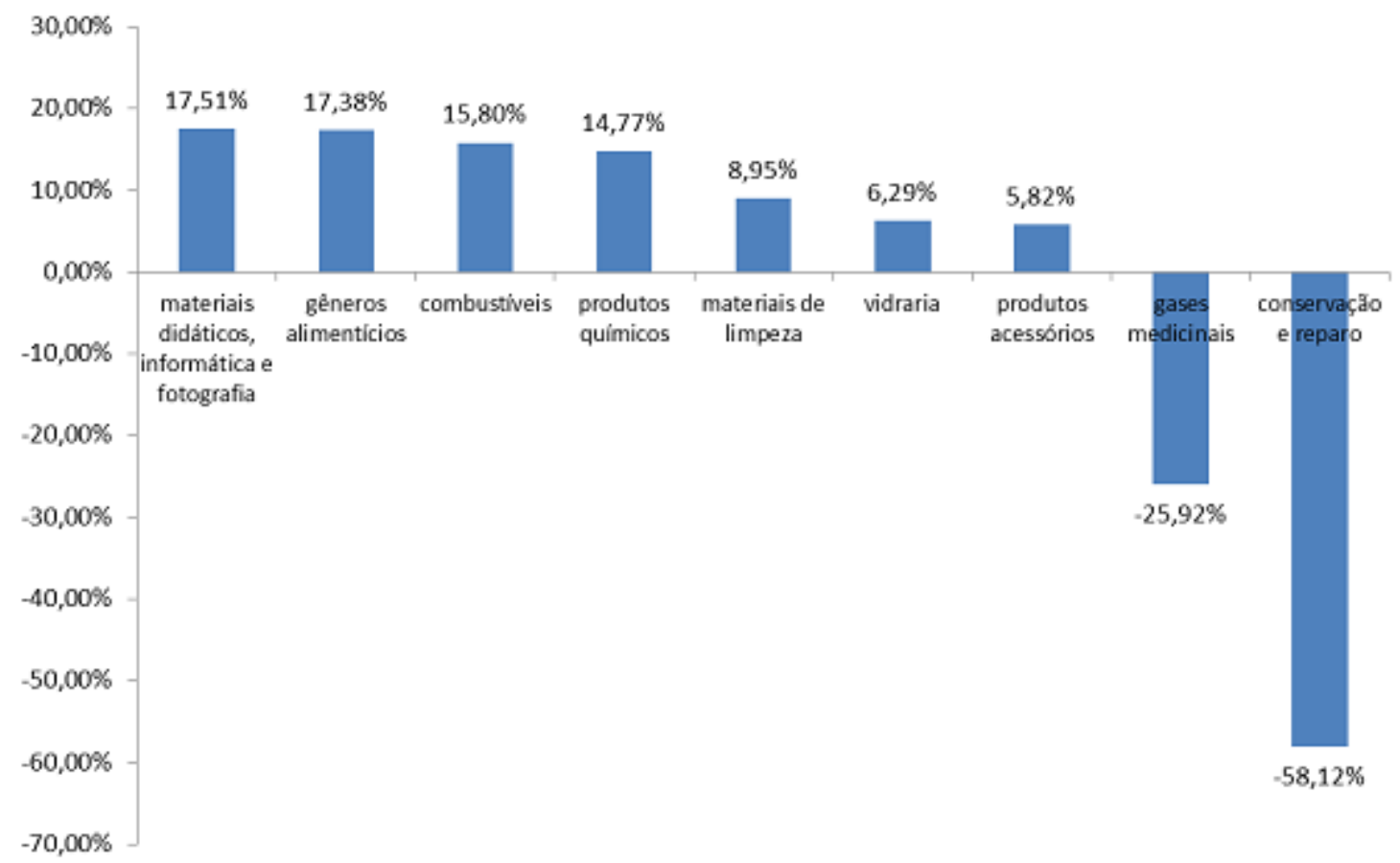

Gráfico 1: IVPH acumulado no ano de 2013 por grupos de materiais de consumo.

A Tabela 1 demonstra o IVPH estratificado por grupos de medicamentos.

Os itens que tiveram maiores contribuições na variação dos preços do grupo medicamentos foram Anfotericina B lipossomal injetável fr $50 \mathrm{mg}$, com variação de 5,43\%; Voriconazol comprimido $200 \mathrm{mg}$, com 3,90\% e I matinib comprimido 400 $\mathrm{mg}$, com $3,87 \%$.

O medicamento Anfotericina B teve aumento de preços de $11,29 \%$ de setembro para outubro/13 com contribuição significativa na composição da variação do grupo pois tem peso forte aliado à variação do preço. O mesmo ocorreu com o medicamento Voriconazol comprimido que teve aumento de preços de 14,22 \% em janeiro de 2013.

\section{DISCUSSÃO}

No gráfico 2 observamos o comparativo entre os IVPH e os índices oficiais no Brasil, no ano de 2013.

Nos dados do ano de 2013, o IPC-FIPE foi de $3,89 \%$, o IGP-M foi de $5,53 \%$ e o ICV-DIEESE foi $6,02 \%$. Os índices obtidos por grupos de ma- teriais de consumo demonstrados no Gráfico 1 mostram que:

- os grupos materiais didáticos, informática e fotografia; gêneros alimentícios; combustíveis; produtos químicos e materiais de limpeza são maiores que os índices oficiais no mesmo período no país.

- os grupos materiais de limpeza, vidraria e produtos e acessórios estão próximos aos índices oficiais.

- os grupos gases medicinais e, conservação e reparos indicam deflação, com índices bem menores que os oficiais.

O índice de variação de preços para medicamentos em 2013 foi 6,73\% conforme demonstrado no Gráfico 2.

Comparando os índices obtidos por grupos de medicamentos com os índices oficiais anteriormente mencionados, temos que os grupos: ginecológicos, analgésicos, antipiréticos e antirreumáticos, contrastes radiológicos, anti-inflamatórios e anestésicos são maiores.

Em uma analogia, citando fatos reais, é comum a indignação com índices oficiais de inflação 
Tabela 1

Índices específicos de variações de preços por grupos de medicamentos - HCRP, 2013.

\begin{tabular}{|c|c|c|}
\hline & Grupos de medicamentos & Variação \\
\hline 1 & ginecológicos & $107,29 \%$ \\
\hline 2 & analgésicos, antipiréticos antirreumáticos & $67,42 \%$ \\
\hline 3 & contrastes radiológicos & $45,40 \%$ \\
\hline 4 & anti-inflamatórios & $42,09 \%$ \\
\hline 5 & anestésicos & $40,59 \%$ \\
\hline 6 & antiparasitários & $18,52 \%$ \\
\hline 7 & antídotos & $17,10 \%$ \\
\hline 8 & soluções em geral & $12,74 \%$ \\
\hline 9 & vitaminas, sais minerais e oligoelementos & $11,79 \%$ \\
\hline 10 & antibióticos quimioterápicos citostáticos & $10,04 \%$ \\
\hline 11 & complementos alimentares & $7,79 \%$ \\
\hline 12 & dermatológicos & $5,01 \%$ \\
\hline 13 & psicolépticos, neurolépticos e psicoanalépticos & $4,75 \%$ \\
\hline 14 & aparelho digestivo & $4,36 \%$ \\
\hline 15 & autonomotrópicos & $3,50 \%$ \\
\hline 16 & outros & $2,07 \%$ \\
\hline 17 & analgésicos narcóticos & $2,03 \%$ \\
\hline 18 & cardiovasculares e diuréticos & $1,50 \%$ \\
\hline 19 & anticonvulsivantes e miorrelaxantes & $1,46 \%$ \\
\hline 20 & aparelho respiratório & $0,02 \%$ \\
\hline 21 & antissépticos e desinfetantes & $-0,13 \%$ \\
\hline 22 & imunizantes & $-0,36 \%$ \\
\hline 23 & oftalmológicos & $-1,23 \%$ \\
\hline 24 & hormônios e derivados & $-1,61 \%$ \\
\hline 25 & otorrinolaringológicos & $-1,87 \%$ \\
\hline 26 & hipnóticos e sedantes & $-7,59 \%$ \\
\hline 27 & anti-histamínicos e anticinetóticos & $-7,78 \%$ \\
\hline 28 & modificadores hemáticos & $-11,48 \%$ \\
\hline
\end{tabular}

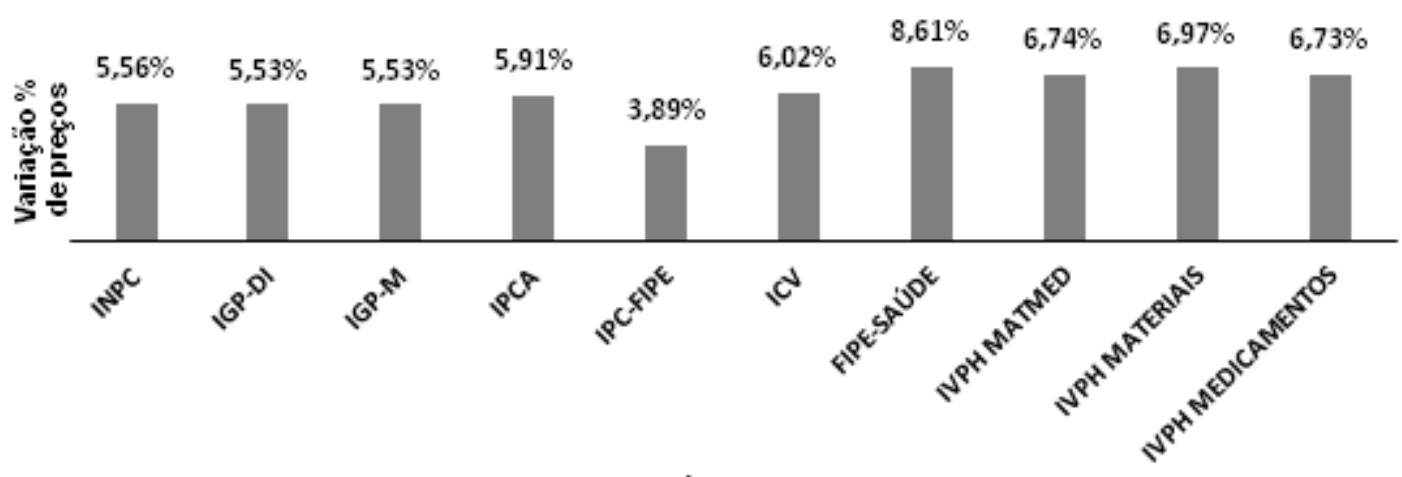

Gráfico 2: Índices de variações de preços oficiais no Brasil, acumulados em 2013 e os IVPH geral e específicos para materiais de consumo e medicamentos em 2013.

Fonte: Banco Central do Brasil e índices desenvolvidos neste estudo. 
de $0,6 \%$ ou $1,2 \%$ quando se declara que houve aumento de $20 \%$, por exemplo, no etanol.

Por isto, não podemos dizer que há inflação quando há aumento de apenas um item ou um grupo de itens na cesta composta.

Inflação é aumento geral de preços e a mensuração da contribuição individual de cada componente ao índice composto é de fundamental importância para verificar quais produtos impactam a variação.

Observou-se que itens de maiores pesos no grupo, ou seja os que tiveram maior influência na cesta pelo seu preço e quantidade, apesar de sofrerem variação de preço em determinado mês ou meses, tiveram pouca variação ou redução de preço em outros meses. Com isto, suas contribuições foram menores do que de outros itens que tiveram peso igual ou inferior no grupo, mas que sofreram variações de preços para maior em diversos meses, vez que a metodologia pondera os pesos por quantidades e preços por mês.

Neste estudo verificou-se que os IVPH gerais foram $6,74 \%$ para materiais e medicamentos, $6,97 \%$ para materiais de consumo e $6,73 \%$ para medicamentos.

Conhecer a variação interna de preços é aspecto importante para o planejamento e acompanhamento das contas de qualquer empresa. No caso específico do setor saúde, os hospitais enfrentam aumento de gastos e de custos tanto pelo aumento de demanda quanto pela incorporação necessária de tecnologias, além das tendências do mercado. ${ }^{24,26}$

Assim, o índice interno de variação de preços pode refletir tendência de mercado, quando estes se aproximam dos índices oficiais, mas também pode ser reflexo do processo interno de compras.

\section{CONCLUSÃO}

Os custos crescentes são realidade marcante na assistência à saúde e tendem impactar na variação do nível de preços do setor resultando em índices maiores do que os gerais de inflação. $21,23,25$
Coerente com esta realidade, este estudo verificou, para o ano 2013, que os IVPH foram $6,74 \%$ para materiais de consumo e medicamentos, $6,97 \%$ específico para materiais de consumo e $6,73 \%$ específico para medicamentos enquanto que os índices oficiais de inflação apontaram os seguintes resultados acumulados no período de janeiro a dezembro de 2013: IPC-FIPE = 3,89\% e IPCA - IBGE = 5,91\%.

Entretanto, os índices IVPH encontrados neste estudo encontram-se menores que o FIPE - SAÚDE, que foi de $8,61 \%$ no mesmo período, sugerindo bom poder de compra da instituição, o que merece investigação mais aprofundada.

Os recursos orçamentários para material de consumo do HCFMRP USP (incluindo medicamentos) advindos do Estado de São Paulo foram de $\mathrm{R} \$ 87.666 .918,00$ em 2013 e $\mathrm{R} \$ 84.347 .956,00 \mathrm{em}$ 2014.27

Corrigido pelo IVPH Geral encontrado de $6,74 \%$, os recursos para 2014 deveriam ser de, no mínimo, R\$93.575.668,27. Entretanto, a situação enfrentada pela instituição foi um recuo no valor orçamentário de 2013 para 2014, de 3,78\%.

Neste mesmo período, entre 2012 e 2013, o movimento hospitalar cresceu $2,5 \%$ nas consultas ambulatoriais, $0,3 \%$ nas internações e $4,7 \%$ nas cirurgias realizadas.

Estes números são expressivos quando considerada a variação de preços interna, de 6,74\% em 2013.

Comprar bem a preços praticados no mercado é aplicar bem os parcos recursos disponíveis.

Portanto, as instituições de saúde não podem prescindir de informações financeiras e econômicas. Recursos bem gerenciados podem render mais e a consequência é melhoria da qualidade e maior cobertura para o sistema de saúde.

Assim, a proposta de criar o índice interno de variação de preços vem contribuir como ferramenta para gestão dos recursos e para a análise da evolução do poder de compra do HCFMRP-USP, assim como os índices padronizados de variação de preço são utilizados para análise do poder de compra da moeda. 


\section{REFERÊNCIAS BIBLIOGRÁFICAS}

1. Morato LS, Ramalho W. Inflação interna: um estudo de caso em uma instituição privada de ensino superior. Rev Eletrônica Produção \& Engenharia, 2008; 1: 107-18.

2. BRASIL. Portaria MS/GM no 373, de 27 de fevereiro de 2002. Norma Operacional da Assistência à Saúde - NOAS-SUS 01/02. Brasília: Ministério da Saúde; 2002.

3. Couttolenc BF, Zucchi P. Gestão de recursos financeiros. São Paulo: Faculdade de Saúde Pública, Universidade de São Paulo; 1998.

4. Creese AL, Parker D. Cost analysis in primary health care: a training manual for programme managers. Geneva: World Health Organization; 1994.

5. Ferraz MB. Dilemas e escolhas do sistema de saúde: economia da saúde ou saúde da economia? Rio de Janeiro: Medbook; 2008.

6. Piola SF, Vianna SM. Economia da saúde: conceitos e contribuição para a gestão da saúde. 3 ed. Brasília: IPEA; 2002.

7. Trindade E. Incorporação de novas tecnologias nos serviços de saúde. Rio de Janeiro: Cad Saúde Pública. 2008; 24: 951-64.

8. World Bank Institute. Management in Health: financing for management in health; 2014.

9. Zucchi P, Del Nero C, Malik AM. Gastos em saúde: Os fatores que agem na demanda e na oferta dos serviços de saúde. Saude Soc. 2000; 9:127-50.

10. Smith PC, Witer SN. Risk-pooling in health care financing: the implications for health system performance; health, nutrition and population discussion paper, Washington: The World Bank; 2004.

11. BRASIL. Ministério da Saúde. Secretaria-Executiva. Secretaria de Atenção à Saúde. Departamento de Economia da Saúde, Investimentos e Desenvolvimento. Glossário temático: economia da saúde. 3. ed. Brasília: Ministério da Saúde; 2012.

12. Ocké-Reis CO, Cardoso SS. A regulamentação dos preços dos planos individuais de saúde. Revista de Economia Política, v.31, n.3, p. 455-470; 2011.

13. Gottret $P$, Schieber $G$. Health financing revisited - a practitioner's guide. Washington. The World Bank; 2006.

14. Roberts $M$ et al. Getting health reform right : a guide to improving performance and equity. Oxford; New York: Oxford University Press, 2004.
15. Viana A L d' Á, Silva HP, Scheffer M. Economia e saúde. In: Martins MA et al. (Org.). Clínica Médica. São Paulo: Manole, 2009: 1: 413-20.

16. Morgan SG. Quantifying components of drug expenditure inflation: the british columbia seniors' drug benefit plan. Health Serv Res. 2002: 37: 1243-66.

17. Grootendorst P, Goldsmith L, Hurley J, O'Brien B, Dolovich L. Financial incentives to dispense low cost drugs: a case study of British Columbia Pharmacare. Hamilton: McMaster University; 1996.

18. Hsiao W, et al. Improving Hong Kong's health care system: why and for whom? Hong Kong: Government Printing Department; 1999.

19. Fixler D, Ginsburg M. Health Care Output and Prices in the Producer Price Index. National Bureau of Economic Research. University of Chicago Press. Disponível em http://www.nber.org/books/cut101-1. Acesso em: 26 Jan, 2015.

20. Fava VL. A precisão dos índices de preços. EconomiA, Brasília, 2007;8:39-63.

21. Antonik LR, Veiga DRC. Taxas de inflação e índice de preços, uma abordagem prática; 2012. Acesso em: 20 jan. 2014.http://www.unifae.br/publicações/ pdf/IIseminario/iniciacaoCiente\% C3\% ADfica

22. Farias AM, Laurencel LC. Números índices. Rio de Janeiro: Universidade Federal Fluminense; 2005.

23. Instituto Brasileiro de Geografia e Estatística (IBGE). Sistema Nacional de Índices de Preços ao Consumidor: métodos de cálculo. Rio de Janeiro: IBGE; 2012.

24. Silva HFS, Souza LO. Cálculo da inflação interna no setor saúde - Um case do Hospital Mãe de Deus. São Leopoldo: Universidade do Vale do Rio dos Sinos; 2002

25. Martins PG. Administração de materiais e recursos patrimoniais. São Paulo: Saraiva; 2001

26. Carmo H. A teoria dos índices de preços e o sistema de metas de inflação no Brasil. São Paulo: Instituto de Pesquisa Econômica Aplicada - Seminário no 23, 2004. Disponível em: <www.econ.fea.usp.br/ seminários/artigos2/ipeset04.pdf>. Acesso em 20 jan. 2015.

27. Hospital das Clínicas da Faculdade de Medicina de Ribeirão Preto da Universidade de São Paulo. Relatório de Atividades. Ribeirão Preto; 2015. 